\title{
Barro-Gordon Revisited: Reputational Equilibria with Inferential Expectations
}

\author{
Timo Henckel \\ Australian National University* \\ Gordon D. Menzies \\ University of Technology Sydney ${ }^{\dagger}$ \\ Nicholas Prokhovnik \\ Daniel J. Zizzo \\ University of Technology Sydney ${ }^{\ddagger} \quad$ University of East Anglia ${ }^{\S}$
}

September 2010

\begin{abstract}
We incorporate inferential expectations into the Barro-Gordon model (1983a) of time inconsistency and consider reputational equilibria. The range of sustainable equilibria shrinks as the private sector becomes more belief-conservative.

Keywords: credibility; time inconsistency; reputation; inferential expectations.

JEL Classification: E52; E61
\end{abstract}

\footnotetext{
${ }^{*}$ Corresponding author. Research School of Economics, Australian National University, Canberra ACT 0200, Australia. (timo.henckel@anu.edu.au). Phone: +61-2-61255540. Fax: +61-261250182 .

${ }^{\dagger}$ University of Technology, PO Box 123, Broadway, Sydney NSW 2007, Australia. (gordon.menzies@uts.edu.au). Phone: +61-2-95147728. Fax: +61-2-95147711.

${ }^{\ddagger}$ University of Technology, PO Box 123, Broadway, Sydney NSW 2007, Australia.

${ }^{\S}$ School of Economics and CBESS, University of East Anglia, Norwich NR4 7TJ, United Kingdom. (d.zizzo@uea.ac.uk).
} 


\section{Introduction}

Barro and Gordon (1983b) famously showed that discretionary policy by a central bank attempting to push output above the natural level delivers a costly average inflation bias. Barro and Gordon (1983a) argued that in a repeated game reputational concerns by the central bank can reduce the inflation bias by sustaining equilibria with inflation rates below the one-shot discretionary equilibrium rate. Their model applies the folk theorem for infinite-horizon repeated games (Fudenberg and Maskin, 1986) in which the private sector follows a grim trigger strategy that punishes the central bank should it renege on its promise to deliver low inflation. ${ }^{1}$

In this paper we refine the Barro-Gordon reputational equilibria by assuming that the inflation process is noisy and by endowing the private sector with inferential expectations (IE), a boundedly-rational model of belief formation based on hypothesis testing, presented and justified in Menzies and Zizzo (2009). Under IE, agents have a null hypothesis $\left(H_{0}\right)$ belief about a variable, which they maintain unless enough evidence exists such that, in a hypothesis test with test size $\alpha$, they can reject it for an alternative hypothesis $\left(H_{1}\right)$. IE can be considered a 'fast and frugal heuristic' (Gigerenzer et al., 1999) of belief formation characterized by information gathering and information processing costs.

We show that when the private sector has inferential expectations and time is used as a test statistic the enforceable range of equilibrium inflation rates shrinks. This is an important finding as it highlights the strong rationality requirement for the sustainability of low inflation rates, an aspect largely unconsidered in the existing literature.

We feel that this kind of setup is much closer to reality. Most central banks with inflation targets have adopted target bands, thus admitting inflation is uncertain and not perfectly controllable. And the assumption of belief conservatism accords with a slew of evidence from psychology and economics. ${ }^{2}$

\footnotetext{
${ }^{1}$ For an overview of models studying reputational equilibria and the associated references see Walsh (2010).

${ }^{2}$ See, for example, Gigerenzer and Murray (1987) and Menzies and Zizzo (2007).
} 


\section{The Model}

\subsection{Setup}

There are two players, the monetary authority and the 'private sector'. The monetary authority's expected intertemporal loss function is

$$
V_{t}=\mathbb{E}_{t}\left[\sum_{s=0}^{\infty} q^{s} L_{s}\right]
$$

where the period loss function is

$$
L_{s}=\frac{a}{2} \pi_{s}^{2}-b\left(\pi_{s}-\pi_{s}^{e}\right), \quad a, b>0,
$$

$q \in(0,1)$ denotes a constant discount factor, and $\mathbb{E}_{t}$ is the mathematical expectations operator, conditional on time $t$ information. The period loss function is standard - the first term captures the nonlinear cost of a deviation of inflation from the socially optimal level, which is normalized to zero. The second term models the benefit from unanticipated inflation as encapsulated in a Lucas supply function. The constant parameters $a$ and $b$ describe the monetary authority's relative weights for inflation stabilization and output expansion, respectively. Since there are no lags in the system the current choice of inflation imposes no direct constraints on future choices, making the optimization problem static.

Inflation is given by

$$
\pi_{t}=\Delta m_{t}+v_{t},
$$

where $\Delta m$ is the growth rate of the money supply - the central bank's policy instrument - and $v \sim\left(0, \sigma^{2}\right)$ denotes a velocity disturbance.

The private sector uses inferential expectations when forming beliefs about the inflation rate $\pi$. Because inflation is not directly under the central bank's control, the private sector does not know whether a deviation of the inflation rate from the announced target is due to a change in the policy instrument or due to a velocity shock.

In some initial period the central bank announces an official inflation target, $\pi^{*}$. Within each period the timing of events is as follows: ${ }^{3}$ First, the private sector sets $\pi^{e}$. Then the central bank chooses its instrument setting $\Delta m$, after which the velocity shock $v$ is realized and actual inflation and output are determined. Finally, the private sector forms its inferential expectations.

\footnotetext{
${ }^{3}$ At the beginning of the period the monetary authority and the private sector share the same information set.
} 


\subsection{Static Equilibria}

Consider first the solutions to the above problem under rational expectations (RE), as described in Barro and Gordon (1983a).

Discretion Minimizing $L_{t}$, taking $\pi_{t}^{e}$ as given, delivers the time consistent, optimal choice of $\Delta m$,

$$
\Delta m^{d}=\frac{b}{a}
$$

where the $d$-superscript denotes discretion. Actual inflation is given by $\pi_{t}=$ $b / a+v_{t}$.

With rational expectations, $\pi_{t}^{e}=\pi^{d}=\Delta m^{d}=b / a$. The period $t$ expected loss to the monetary authority becomes

$$
\mathbb{E}_{t}\left[L_{t}^{d}\right]=\frac{a}{2}\left(\left(\frac{b}{a}\right)^{2}+\sigma^{2}\right) .
$$

Commitment If the policy maker can commit to an announced policy target, $\pi^{*}$, and the private sector believes this announcement, the latter's expectations become endogenous, implying $\pi_{t}^{e}=\pi^{*}$. The associated policy setting is

$$
\Delta m^{*}=\pi^{*}
$$

with an expected loss of

$$
\mathbb{E}_{t}\left[L_{t}^{c}\right]=\frac{a}{2}\left(\left(\pi^{*}\right)^{2}+\sigma^{2}\right),
$$

which is minimized when $\pi^{*}=\Delta m^{*}=0$.

Cheating If the private sector believes an announced rule, the policy maker has an incentive to renege on its announcement (cheat). Suppose the monetary authority announced an inflation target of $\pi^{*}$, implying $\Delta m^{*}=\pi^{*}$, which is believed by the private sector: $\pi^{e}=\pi^{*}=\Delta m^{*}$. The monetary authority's optimal choice of $\Delta m_{t}$ is the discretionary solution,

$$
\Delta m^{c h}=\frac{b}{a},
$$


where the $c h$-superscript denotes cheating. The associated expected loss is

$$
\mathbb{E}_{t}\left[L_{t}^{c h}\right]=\frac{a}{2}\left(-\left(\frac{b}{a}\right)^{2}+\sigma^{2}+\frac{2 b}{a} \pi^{*}\right) .
$$

Quick inspection reveals that, if $\pi^{*}<b / a, \mathbb{E}_{t}\left[L_{t}^{d}\right]>\mathbb{E}_{t}\left[L_{t}^{c}\right]>\mathbb{E}_{t}\left[L_{t}^{c h}\right]$, replicating the conventional result that commitment is better than discretion.

\subsection{Sustainable Reputational Equilibria}

In an infinitely repeated game, a reputational equilibrium offers a possible solution to the costly inflation bias resulting from myopic discretion, as shown by Barro and Gordon (1983a).

\subsubsection{Grim Trigger Strategy with Inferential Expectations}

Assume that the private sector follows a grim trigger strategy, as in Friedman (1971), when forming inferential expectations. Due to noise in the inflation process the private sector each period must decide whether the monetary authority is obeying its announced policy rule or cheating. This decision is the outcome of a statistical hypothesis test with the following null and alternative hypotheses:

$$
\begin{aligned}
& H_{0}: \Delta m=\pi^{*}=\Delta m^{*} \text { (monetary authority obeys rule) } \\
& H_{1}: \Delta m>\pi^{*}=\Delta m^{*} \text { (monetary authority cheated) }
\end{aligned}
$$

The signal is the number of periods for which $\pi>\pi^{*}$ while the test statistic is $\tau$, based on a sampling distribution discussed below.

Inflation expectations at the end of peoriod $t-1$ are revised according to the outcome of the hypothesis test:

$$
\pi_{t}^{e}=\left\{\begin{array}{cc}
\pi^{*}=\Delta m^{*} & \text { if } H_{0} \text { accepted } \\
\pi^{d}=\Delta m^{d}=b / a & \text { if } H_{1} \text { accepted }
\end{array}\right.
$$

Without loss of generality, we assume that the private sector's punishment (setting $\pi^{e}=\pi^{d}=\Delta m^{d}$ if $H_{1}$ accepted) lasts one period only. After the punishment phase history is erased - the counter for the test statistic reverts to zero and $\pi^{e}=\pi^{*}$.

For simplicity we rule out central bank strategies that are contingent on the test statistic $\tau$. In other words, the central bank does not attempt to exploit the private sector's belief conservatism. 


\subsubsection{Benefits and Costs}

The benefit of cheating (temptation) in period $t$ is the difference between the expected utility loss of a commitment policy and a cheating policy. For a given target inflation rate $\pi^{*}$ the benefit to cheating in period $t$ is

$$
\sum_{i=0}^{n} q^{i}\left(\mathbb{E}_{t}\left[L_{t+i}^{c}\right]-\mathbb{E}_{t}\left[L_{t+i}^{c h}\right]\right)=A Q
$$

where $n$ denotes the period in which private agents decide the monetary authority is cheating and $A$ and $Q$ are defined as ${ }^{4}$

$$
\begin{aligned}
A & \equiv \frac{a}{2}\left(\frac{b}{a}-\pi^{*}\right)^{2}, \\
Q & \equiv \frac{1-q^{n}}{1-q}
\end{aligned}
$$

The greater the distance between $\pi^{*}$ and $b / a$, the greater the temptation to cheat. At $\pi^{*}=b / a$, there is no temptation to cheat because $b / a$ is already the optimal inflation rate under discretion. The benefit of adhering to the announced policy rule (enforcement) is given by

$$
q^{n}\left(\mathbb{E}_{t}\left[L_{t+n}^{d}\right]-\mathbb{E}_{t}\left[L_{t+n}^{c}\right]\right)=q^{n}\left[\frac{a}{2}\left(\left(\frac{b}{a}\right)^{2}-\left(\pi^{*}\right)^{2}\right)\right],
$$

which is the cost, in present value terms, of reverting to discretionary policy in period $n$. For values of $\pi^{*}$ below and above $b / a$, an increase in $\pi^{*}$ reduces the cost of cheating at an increasing rate.

\subsubsection{Range of Reputational Equilibria}

For a rule to be enforceable the benefit to cheating (temptation) must be less than or equal to the cost of cheating (enforcement).

Figure 1 shows the temptation and enforcement curves. The bounds of the range of enforceable (time consistent) inflation rates are determined by the points where the temptation and enforcement curves intersect. Setting (10) equal to (11)

\footnotetext{
${ }^{4}$ Note that $L_{t+i}^{c}-L_{t+i}^{c h}=L_{t}^{c}-L_{t}^{c h}$.
} 
and solving for both roots of $\pi^{*}$, yields the lower bound $\left(\pi_{1}^{*}\right)$ and upper bound $\left(\pi_{2}^{*}\right)$ of the range:

$$
\left[\frac{b\left(Q-q^{n}\right)}{a\left(Q+q^{n}\right)}, \frac{b}{a}\right] .
$$

An optimizing policy maker will pick the value from within the enforceable range that minimizes its loss, which is simply the lower bound.

\subsubsection{Maximum Length of Cheating}

Based on (3), for a given $\pi^{*}=\Delta m^{*}$, actual inflation is just as likely to be above $\pi^{*}$ as below $\pi^{*}$. When observed inflation exceeds $\pi^{*}$ for successive periods, the probability that the monetary authority has not cheated decreases over time.

More formally, assume a discrete probability density function given by

$$
f(\tau)=\left(\frac{1}{2}\right)^{\tau}, \quad \tau=1,2,3, \ldots,
$$

which gives the probability that $\pi>\pi^{*}$ for $\tau$ successive periods if $\Delta m=\Delta m^{*}$. The $p$-value is

$$
p=\sum_{\tau=n}^{\infty} f(\tau)=\sum_{\tau=n}^{\infty} 2^{-\tau}=2^{1-n} .
$$

$H_{0}$ is rejected if

$$
p=2^{1-n} \leq \alpha,
$$

which, when solved for $n$, becomes

$$
n \geq 1-\frac{\ln \alpha}{\ln 2}
$$

Evidently, the lower bound of the enforceable range and consequently the range itself becomes a function of $\alpha$ :

$$
\pi_{1}^{*}=\frac{b\left(Q-q^{1-(\ln \alpha / \ln 2)}\right)}{a\left(Q+q^{1-(\ln \alpha / \ln 2)}\right)}
$$

with

$$
Q=\frac{1-q^{1-(\ln \alpha / \ln 2)}}{1-q}
$$




\subsubsection{Comparison with Original Barro-Gordon}

Comparing this model to the original Barro-Gordon model reveals that the upper bound of the enforceable range, $\pi_{2}^{*}=b / a$, is the same in both cases, while $\pi_{1}^{*}$ in the IE-model is a function of $n$, the number of periods actual inflation exceeds the target. The $y$-intercept of the temptation curve in figure $1\left(Q\left(b^{2} / 2 a\right)\right)$ is increasing in $n$ while the $y$-intercept of the enforcement curve $\left(q^{n}\left(b^{2} / 2 a\right)\right)$ is decreasing in $n$. Hence, as the number of periods during which inflation is allowed to exceed the target increases, the enforceable range shrinks.

Two extremes are useful to consider. First, consider the case where $n$ approaches infinity. The lower bound of the range is thus

$$
\pi_{1}^{*}=\lim _{n \rightarrow \infty} \frac{b\left(Q-q^{n}\right)}{a\left(Q+q^{n}\right)}=\lim _{n \rightarrow \infty} \frac{b}{a} \frac{\left(\lim _{n \rightarrow \infty} Q-0\right)}{\left(\lim _{n \rightarrow \infty} Q+0\right)}=\frac{b}{a}=\pi_{2}^{*} .
$$

Hence, the length of the enforceable range collapses to zero and the only enforceable inflation rate is $b / a$.

Now consider the case where $n=1$. In this case the lower bound is

$$
\pi_{1}^{*}=\frac{b\left(Q-q^{n}\right)}{a\left(Q+q^{n}\right)}=\frac{b(1-q)}{a(1+q)}
$$

which simply equals the lower bound in the original Barro-Gordon model. The latter model is therefore nested in the model with inferential expectations. ${ }^{5}$

\section{Discussion}

When agents have inferential expectations in the Barro-Gordon model the enforceable range of equilibrium inflation rates narrows. Because the inflation process is noisy, the private sector is unable to decipher whether an increase in observed inflation is due to a velocity shock or to systematic expansionary policy. If the private sector is belief-conservative it requires a large amount of information (several periods in which $\pi>\pi^{*}$ ) before it changes its belief. The monetary authority is thus able to cheat for several periods before being 'caught' and so the temptation to cheat grows. The private sector recognizes this and responds by tolerating a smaller range of inflation targets. Consequently, the enforceable range depends on

\footnotetext{
${ }^{5}$ When $n=1, \alpha=1$. In other words, if the private sector possesses no belief-conservatism $(\alpha=1)$, inflation cannot exceed the official target for longer than one period.
} 
the test size $\alpha$ used in the private sector's hypothesis tests. The original BarroGordon model is a special case of the model with inferential expectations in which $\alpha=1$.

\section{References}

[1] Barro, R. J., Gordon, D. B., 1983a. Rules, discretion and reputation in a model of monetary policy, Journal of Monetary Economics, 12, 101-121.

[2] R. J., Gordon, D. B., 1983b. A positive theory of monetary policy in a naturalrate model, Journal of Political Economy, 91, 589-610.

[3] Friedman, J. W., 1971. A non-cooperative equilibrium for supergames, Review of Economic Studies, 38, 861-874.

[4] Fudenberg, D., Maskin, E., 1986. Folk theorems for repeated games with discounting or with incomplete information, Econometrica, 54, 533-554.

[5] Gigerenzer, G., Murray, D. J., 1987. Cognition as Intuitive Statistics. Lawrence Erlbaum, London.

[6] Gigerenzer, G., Todd, P. M., the ABC Research Group, 1999. Simple Heuristics That Make Us Smart. Oxford University Press, Oxford.

[7] Menzies, G. D., Zizzo, D. J. 2007. Exchange rate markets and conservative inferential expectations, CAMA Working Paper 2/2007.

[8] Menzies, G. D., Zizzo, D. J., 2009. Inferential expectations, The B.E. Journal of Macroeconomics (Advances), 9, Article 42.

[9] Walsh, C. E., 2010. Monetary Theory and Policy, 3rd edition. MIT Press, Cambridge. 


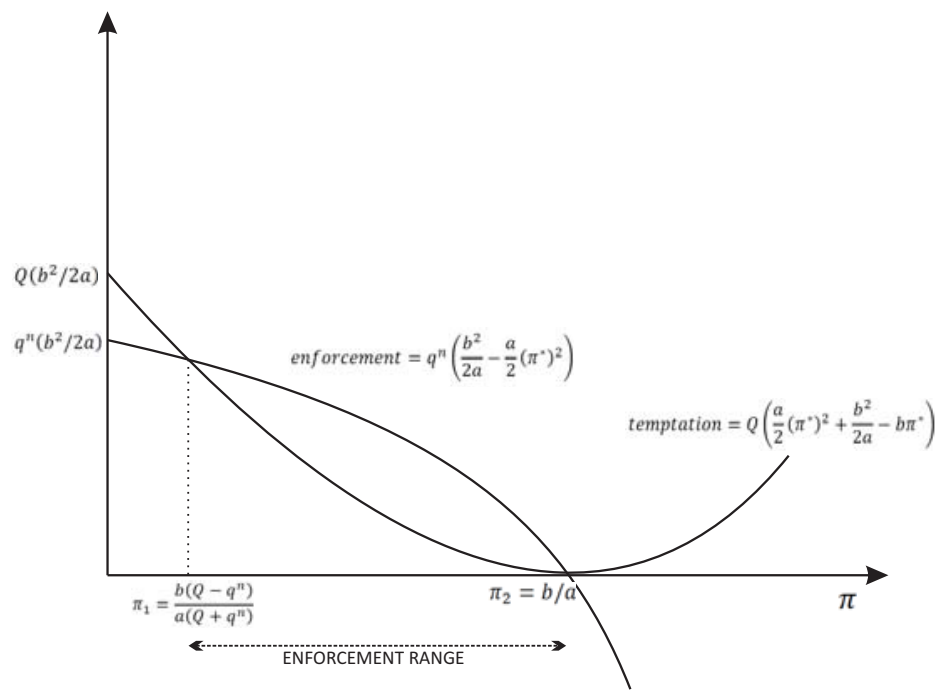

Figure 1: Range of enforceable inflation rates 